\title{
State of the art online monitoring system for the waste beam in the rapid cycling synchrotron of the Japan Proton Accelerator Research Complex
}

\author{
P. K. Saha, ${ }^{*}$ S. Hatakeyama, K. Yamamoto, M. Yoshimoto, H. Harada, H. Hotchi, Y. Shobuda, and M. Kinsho \\ Japan Atomic Energy Agency (JAEA), Tokai, Ibaraki 319-1195, Japan \\ K. Satou and Y. Irie \\ High Energy Accelerator Research Organization (KEK), Tsukuba, Ibaraki 305-0801, Japan \\ (Received 16 May 2011; published 5 July 2011)
}

\begin{abstract}
The $3 \mathrm{GeV}$ rapid cycling synchrotron (RCS) at Japan Proton Accelerator Research Complex is already in user operation stage, delivering a stable and relatively high power beam to the Material and Life Science Experimental Facility as well as to the main ring. RCS utilizes multiturn $\mathrm{H}^{-}$charge-exchange injection for which a hybrid-type boron doped carbon stripper foil known as $\mathrm{HBC}$ foil with a thickness of around $200 \mu \mathrm{g} / \mathrm{cm}^{2}$ is used at the present injection beam energy of $181 \mathrm{MeV}$. It will be changed to $290 \mu \mathrm{g} / \mathrm{cm}^{2}$ at the near future upgrade and design injection beam energy of $400 \mathrm{MeV}$. The stripping efficiencies are expected to be $99.6 \%$ and $99.7 \%$ in the present and the later case, respectively. The remaining $0.4 \%$ or $0.3 \%$ of the beam is called the waste beam and is transported to the injection beam dump with a capacity of only $4 \mathrm{~kW}$. The full power of the injected beam at $400 \mathrm{MeV}$ is $133 \mathrm{~kW}$, while the waste beam power is calculated to be about $0.4 \mathrm{~kW}$. The raw signal measured by a current transformer placed downstream of the waste beam dump line contains a large noise and it is practically very hard to identify the beam signal. However, a fast Fourier transformation analysis of the raw signal made it possible to clearly identify the beam signal. The power spectrum is calibrated with respect to the known injected beam current. The absolute error of the monitor system is $0.2 \mathrm{mV}$, which gives the ratio of the waste beam to the injected beam as $(0.38 \pm 0.03) \%$. Such an accurate and realtime monitoring system provides quite an efficient method to know the conditions of the stripper foil and the incoming linac beam during the user operation.
\end{abstract}

DOI: 10.1103/PhysRevSTAB.14.072801

PACS numbers: 29.20.dk, 29.27.Ac

\section{INTRODUCTION}

The $3 \mathrm{GeV}$ rapid cycling synchrotron (RCS) at Japan Proton Accelerator Research Complex (J-PARC) is now in user operation stage, delivering a stable and high power beam of $200 \mathrm{~kW}$ to the neutron target in the Material and Life Science Experimental Facility (MLF) as well as an equivalent beam power of more than $250 \mathrm{~kW}$ to the main ring (MR). In addition, achievement of a beam power of $300 \mathrm{~kW}$ has already been demonstrated for about one hour of operation [1]. The design goal with the RCS is to achieve $1 \mathrm{MW}$ of output beam power with injection and extraction energies of 0.4 and $3 \mathrm{GeV}$, respectively, having $8.3 \times 10^{13}$ protons per pulse at a repetition rate of $25 \mathrm{~Hz}$ [2]. However, the injection energy at present is $181 \mathrm{MeV}$. Then with the same extraction energy of $3 \mathrm{GeV}$, depending on the linac beam intensity, a maximum output beam power of around $0.6 \mathrm{MW}$ is expected.

\footnotetext{
*Corresponding author. saha.pranab@j-parc.jp
}

Published by the American Physical Society under the terms of the Creative Commons Attribution 3.0 License. Further distribution of this work must maintain attribution to the author(s) and the published article's title, journal citation, and DOI.
In order to increase the number of circulating protons, RCS utilizes the multiturn $\mathrm{H}^{-}$charge-exchange injection technique during the injection period of $0.5 \mathrm{~ms}$. The incoming $\mathrm{H}^{-}$beam from linac is converted to a proton beam by using the primary stripper foil named the 1 st foil placed in the middle of four bump magnets as shown in Fig. 1 [3]. The primary stripper foil used in the RCS is a so-called double-layer type of the hybrid-type boron doped carbon (HBC) foil [4]. The two electrons of $\mathrm{H}^{-}$are detached as efficiently as possible so as to achieve a stripping efficiency as high as $\sim 100 \%$. The design thickness of the primary stripper foil at the present $181 \mathrm{MeV}$ injection is $200 \mu \mathrm{g} / \mathrm{cm}^{2}$. By using the past experimental data, the stripping efficiency is calculated to be $99.6 \%$ [5]. The primary stripper foil will be changed to a thickness of $290 \mu \mathrm{g} / \mathrm{cm}^{2}$ at the upgraded injection energy of $400 \mathrm{MeV}$, where the stripping efficiency is expected to be $99.7 \%$, slightly better than the present one [6]. The remaining $0.4 \%$ or $0.3 \%$ of the unstripped beam is mostly partially stripped particles (single electron detachment) and thus it is neutral (uncharged) and is called $\mathrm{H}^{0}$ beam. They are further stripped to a proton beam by the secondary stripper foil (named 2nd foil) placed about $2 \mathrm{~m}$ downstream from the primary stripper foil and then transported to the injection beam dump. There is also one more secondary stripper 


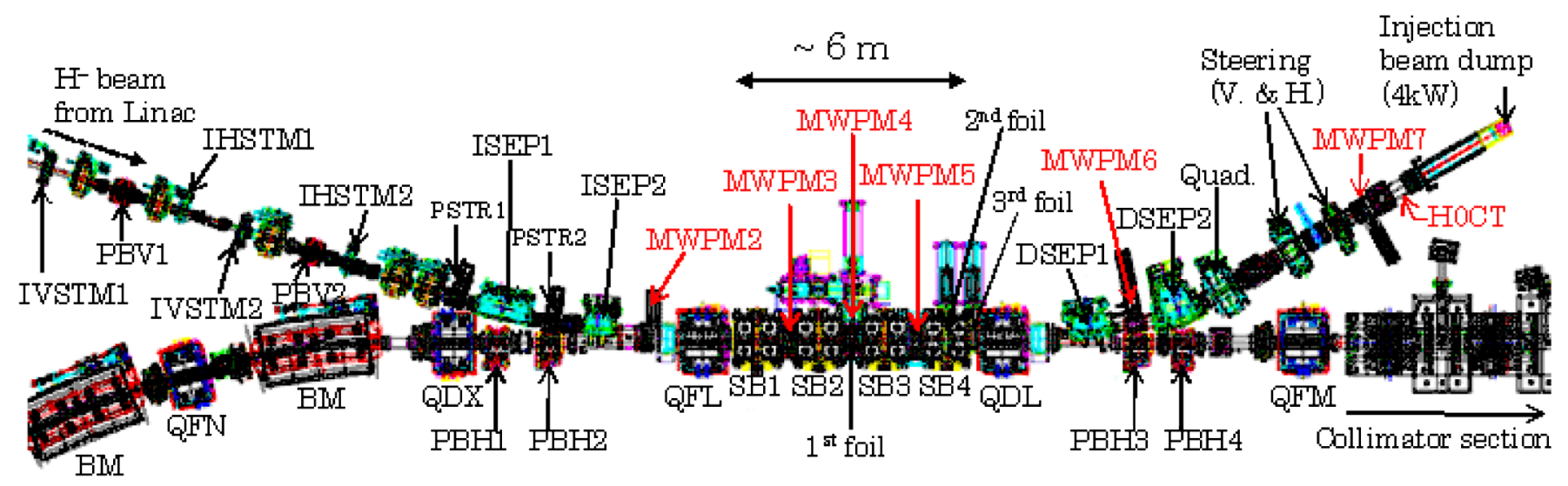

FIG. 1. Layout of RCS injection and successive H0 dump line. The primary stripper foil named as the 1st foil is placed in the middle of four bump magnets (SB1-4). The partially stripped $\mathrm{H}^{0}$ and unstripped $\mathrm{H}^{-}$beams (if any) are stripped to proton beams by two secondary stripper foils, namely, the 2 nd and 3rd foils, respectively. They are called waste beam and are transported to the injection beam dump. A current transformer (CT) named H0CT is placed at the end of the dump and is mainly used as a personal protection system (PPS) for the RCS operation. In order to measure as well as for online monitoring of the waste beam at the dump, a signal from the HOCT is used for the present study.

foil named 3rd foil placed at the end of the bump magnets. It is used for stripping any unstripped $\mathrm{H}^{-}$beam if it were remaining after the 1st foil due to unexpected long tail or halo of the incoming beam, pin holes in the 1st foil, degradation of the 1st foil thickness, magnet failure, and any other possible occurrence by which injected beam misses the 1st foil. They are also transported to the injection beam dump. The secondary stripper foils are thicker $\left(500 \mu \mathrm{g} / \mathrm{cm}^{2}\right)$ than the primary one and thus the stripping efficiencies are expected to be almost 100\% (99.9999\% [5]). As in the ideal case, the main component of the waste beam is $\mathrm{H}^{0}$, the injection dump is also called the $\mathrm{H} 0$ dump.

The thickness of the presently used HBC primary stripper foil is close to the design value and is estimated to be $198 \mu \mathrm{g} / \mathrm{cm}^{2}$. The stripping efficiency is thus expected to be almost the same as designed and is about 99.6\% [5]. However, a foil, especially the primary one with a stripping efficiency of $100 \%$, is quite impossible to use in reality. If a much thicker foil were needed, then the uncontrolled beam loss due to the foil scattering as the circulating beam hits the foil during multiturn injection would increase significantly near the injection area [7]. A thinner foil, on the other hand, makes an increase of the waste beam in the injection dump and thus a higher capacity dump would then be needed, which is both space and money consuming. Proper maintenance of a higher capacity beam dump is also one of the considerable concerns. The present injection dump has a capacity of $4 \mathrm{~kW}$, where the design waste beam power with all design parameters at the near future $400 \mathrm{MeV}$ injection is expected to be $0.4 \mathrm{~kW}(0.3 \%$ of $133 \mathrm{~kW}$ injected beam). It is very important to monitor the beam current in the $\mathrm{H} 0$ dump as failures can happen any time during operation. A foil has a certain lifetime and usually this lifetime becomes shorter as beam intensity goes higher. A proper monitoring system of the waste beam is then necessary even for checking the degradation of the stripper foil thickness in a high power machine.

However, not only because of a small fraction of $0.3 \%-0.4 \%$ of the beam but also due to large noise from the nearby complicated injection system, it is very hard to measure the signal clearly and thus usually one needs a sophisticated monitoring system. There had been a lot of discussions and some efforts in the design stage to establish a system for monitoring the waste beam current in the injection beam dump [8] but unfortunately due to the expense and space consumption a sophisticated monitoring system has not yet been realized. It would be hard to adopt in the present beam line configuration concerning space.

However, we have continued our efforts for possible ways to monitor such a low fraction of the waste beam in the injection beam dump. As a result, a rather precise method has been recently established, which employs a simple principle and does not need any sophisticated device or monitor. The signal measured by an already placed current transformer (CT) named H0CT (see Fig. 1) is first collected by an oscilloscope and then a fast Fourier transformation (FFT) analysis is done. As a result, picking up the amplitude of the power spectrum corresponding to the frequency of the intermediate pulse, which depends on the frequency of the RCS rf system, gives the beam signal [9]. The intermediate pulse usually with a width of $600 \mathrm{~ns}$ is formed by chopping the micropulses of the linac beam, while the RCS rf period at injection is $1066 \mathrm{~ns}$. The ratio of such a signal measured in the normal operation to that measured by transporting all of the injected beam to the dump simply gives the fraction of the waste beam in the injection beam dump. The coincidence of the same frequency from any other source, if any, can be easily checked 
by the spectrum with no beam and thus one can be confident with the measurement.

In order to check the consistency of the measurement, a systematic study was performed. As the thickness of the stripper foil is known, the stripping efficiency of the given foil is measured in the present framework. The experiment was done with a rather large size foil and beam positioning in the foil was well optimized so as to cover the injected beam completely in the foil. As a result, only the partially stripped $\mathrm{H}^{0}$ component of the beam was thus expected in the injection beam dump because of a negligibly smaller quantity of the unstripped $\mathrm{H}^{-}$unless there were pinholes in the foil. However, it was also possible to measure each component of the waste beam separately through combination of the secondary stripper foils as explained in detail in the next section. Data was then taken with several experimental conditions and from the offline analysis a precise value of the measured stripping efficiency was given, which was found to be very consistent with the expectation. Through such a precise measurement of the waste beam in the injection beam dump, the present idea is being used for online monitoring of the waste beam during RCS operation.

\section{HOCT AND THE EXPERIMENTAL SETUP}

Figure 2 shows a sketch of the H0CT prepared here in our laboratory. One of the main purposes of this CT is the usage as a personal protection system (PPS) interlock through two amplification steps of the original signal. It is used to check that the (waste) beam power in the $\mathrm{HO}$ dump does not exceed from the design limit. The system makes an alarm if one hour integrated HOCT signal reaches close to the setting threshold. Usually, beam is then stopped by the operator. Otherwise, the beam goes automatically stopped if the integrated beam signal crosses the setting threshold. However, the signal contains a large noise and thus the integrated signal has a large error. As a result, the threshold needs to be set with some margin. The noise floor also varies with the RCS operational condition, and thus one needs to set different calibration

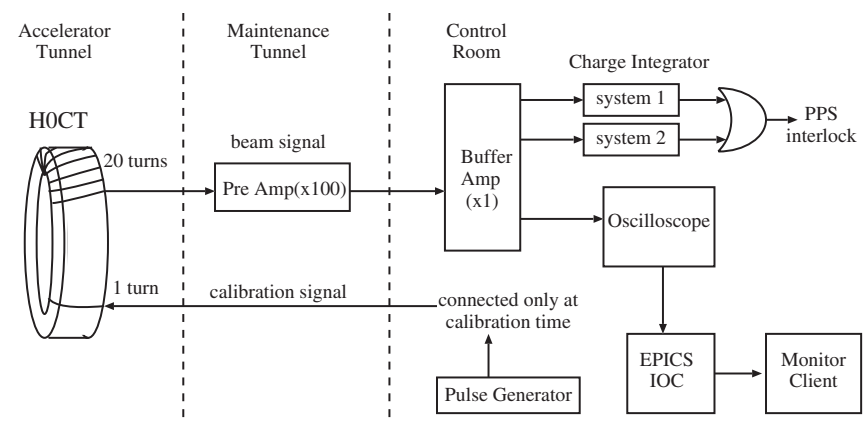

FIG. 2. A sketch of the HOCT and data taking logic. In the present measurement, a two stage amplified signal was fed to an oscilloscope which was online controllable by program. parameters from time to time. In our present approach, we first fed the amplified and buffered signal into an oscilloscope, which is controlled by an operation interface (OPI). The main parameters of the oscilloscope can be controlled online by the OPI. As a result, data can be taken as desired and averaging the signal before FFT analysis sufficiently cancels out the random noise. During MLF user run, an average of 1024 injections is made first and then an FFT analysis is performed by using the Blackmann window function. As RCS runs with $25 \mathrm{~Hz}$, the required time for one data point is about $40 \mathrm{~s}$.

The oscilloscope was used with a DC coupling and high resolution mode. Data was taken with a sampling rate of $100 \mathrm{MS} / \mathrm{s}$, while the horizontal scale of the oscilloscope was set to $50 \mu \mathrm{s} / \mathrm{div}$. A total of 50000 data points for the maximum injection period of $500 \mu$ s were then obtained. The vertical scale of the oscilloscope was set to $1 \mathrm{~V} / \mathrm{div}$. The total number of bins of the power spectrum was 65536 and a frequency resolution of $1.5258 \mathrm{KHz}$ was thus obtained. As for the vertical resolution, the statistical error was reduced as much as possible by taking an average of the time domain signal before the FFT analysis. The measurement error of the waste beam was obtained to be as good as $0.03 \%$.

It is also important to mention that prior to the measurement several fundamental checks of the apparatus as well calibration data were taken. For example, the gain of the preamplifier set at the maintenance tunnel was checked by comparing the signal height before and after the preamplifier and was found to be 100 as expected. An input signal with a rectangular shape was generated by a pulse generator, while the output signal before and after the preamplifier were measured by an oscilloscope. The linearity of the CT was also checked. A pulsed waveform with the same chopping width of $600 \mathrm{~ns}$ as in the real machine was generated by a pulse generator and fed to the oscilloscope. The pulse height of the input signal was varied from a fraction of $\mathrm{mV}$ to several hundred $\mathrm{mV}$, where an average of 1024 shots for each setting was taken. The fundamental frequency of the power spectrum corresponding to the frequency of the minipulses generated by the pulse generator was found to be linear with respect to the heights of the input signals. The linearity of the H0CT with beam was checked also by using a beam with an intensity range of a very minimum to the presently available maximum of $100 \%$ at the $\mathrm{H} 0$ dump. The RCS repetition was set to be $1 \mathrm{~Hz}$. The linearity with using beam was also found to be very good as presented in the next section.

In order to measure the total fraction of the waste beam at the injection dump, experimental data need to be taken with at least two operational modes of the RCS. Namely, (a) injection beam dump mode also called H0 dump mode and (b) the single-pass extraction mode also called one-third mode or (c) the circulating mode [10]. The H0CT signals by the later two modes [(b) and (c)] should 


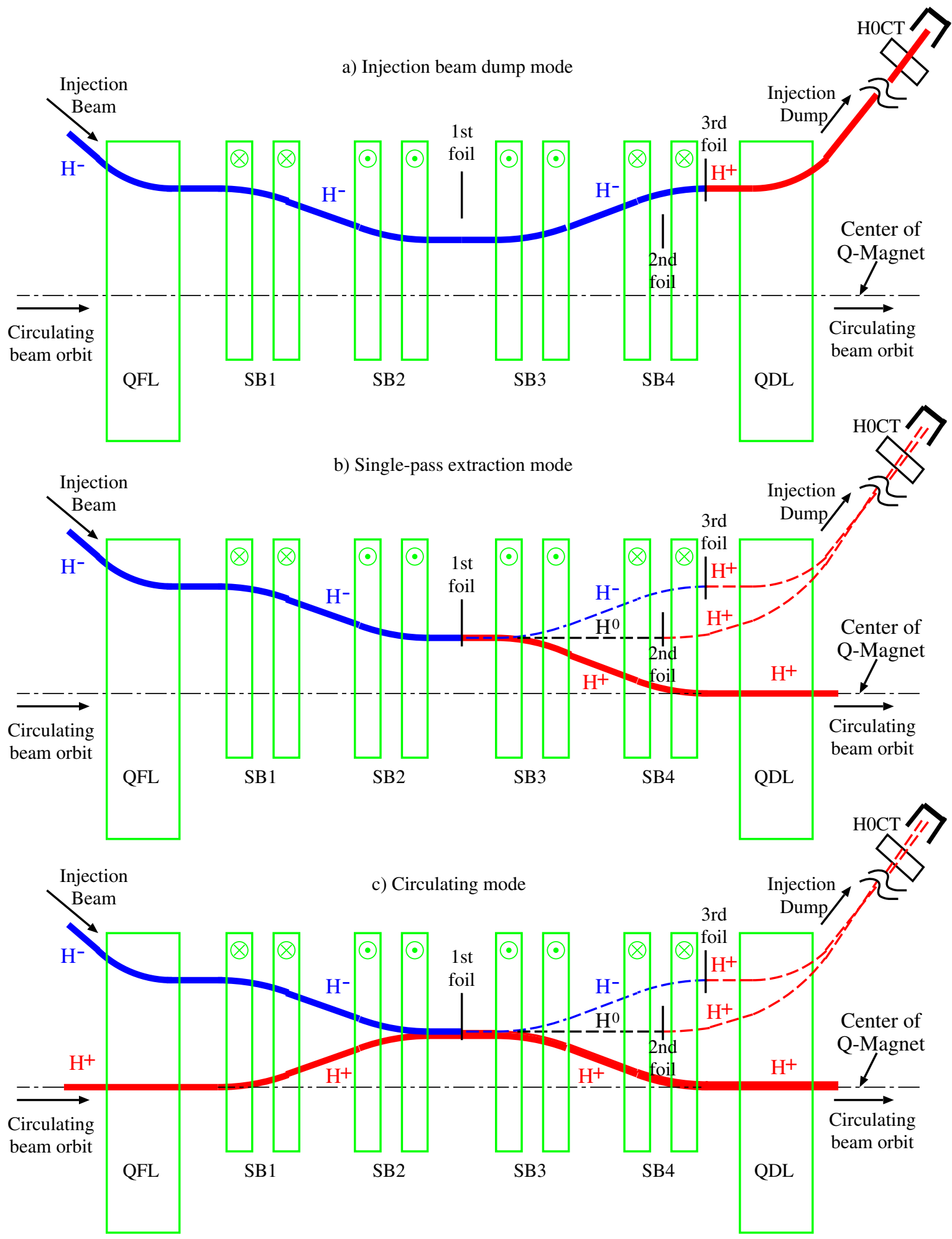

FIG. 3. Roles of the stripper foils and positioning for corresponding operation mode in the RCS. In the injection beam dump mode (a), the primary stripper foil is kept out and thus all the incoming $\mathrm{H}^{-}$beam is stripped at the 3rd stripper foil and is transported to the injection beam dump. However, in the other two modes [(b) and (c)] the primary stripper foil is placed at the right position for the beam injection into the RCS ring through stripping most of the incoming $\mathrm{H}^{-}$beam to $\mathrm{H}^{+}$beam. The waste beams then in both cases are mainly the partially stripped $\mathrm{H}^{0}$ and unstripped $\mathrm{H}^{-}$(if any) remaining at the primary stripper foil and through secondary stripping at the 2nd and 3rd foils, respectively, they are transported to the injection beam dump. A ratio of the H0CT signal with any of two later modes [(b) or (c)] to the former mode (a) gives the fraction of the total waste beam measured at the injection beam dump. 
essentially be the same as only unstripped beam goes to the HO dump during the injection period. For simplicity, data for the first two modes are thus used for the present measurement. The corresponding beam orbits with positioning of stripper foils are shown in Fig. 3.

In the injection beam dump mode, the 1 st foil is removed and thus all of the incoming $\mathrm{H}^{-}$beam is stripped to proton beam by using the $3 \mathrm{rd}$ foil and driven to the injection beam dump, whereas in the single-pass extraction mode the 1st foil is put in the right position so that most of the incoming $\mathrm{H}^{-}$beam is stripped to proton beam and is injected into the RCS ring but travels only one-third of the RCS circumference before being driven to the extraction beam dump [10]. In the former mode, the signal of the H0CT corresponds to a total of the injected beam, whereas in the later mode it corresponds to that only with waste beam [mainly the partial stripped $\mathrm{H}^{0}$ and unstripped $\mathrm{H}^{-}$(if any)] left at the primary stripper foil. A ratio of the signal in the later mode to the former mode thus gives the total fraction of the waste beam at the $\mathrm{H} 0$ dump. If the injected beam is fully covered by an ideal foil with a design thickness of $198 \mu \mathrm{g} / \mathrm{cm}^{2}$, the waste beam at the H0 dump is almost the partially stripped $\mathrm{H}^{0}$ one of $\sim 0.4 \%$ [5]. However, it is also possible to separately measure the unstripped $\mathrm{H}^{-}$(if any) and $\mathrm{H}^{0}$ components by the removal of 2 nd and $3 \mathrm{rd}$ foil, respectively, as can be seen in Fig. 3(b) or Fig. 3(c). Removal of the 2 nd foil means partially stripped $\mathrm{H}^{0}$ beam remains as it is as no secondary stripping to a proton beam at the 2nd foil occurs, and in that case $\mathrm{H}^{0}$ beam cannot be transported to the $\mathrm{H} 0$ dump. Such a $\mathrm{H}^{0}$ beam goes straight and hits the beam chamber at the H0 branch located a little downstream of the quadrupole magnet named QDL (see Fig. 1). The beam at the H0 dump is then left only with an unstripped $\mathrm{H}^{-}$beam (if any) at 1st foil. A similar situation appears with the unstripped $\mathrm{H}^{-}$beam when the 3rd foil is removed and thus beam at the HO dump is then left only with partially stripped $\mathrm{H}^{0}$ beam. In such a way, a fraction of the partially stripped $\mathrm{H}^{0}$ and unstripped $\mathrm{H}^{-}$ beam left at the primary stripper foil can then be measured separately. As a result, the thickness of the primary stripper foil can also then be investigated precisely by using previous experimental measurements [5].

Figure 4 shows the dimension of the primary stripper foil plotted together with the size and positioning of the injected beam set for the present measurement in the singlepass extraction mode. In order to measure a realistic fraction of the waste beam at the injection dump, the foil edge was set as far as $25 \mathrm{~mm}$ from the injected beam center. It was done in order to make sure there is no injected beam missing the foil. Thus, there was only a realistic fraction of the waste beam at the H0 dump (assume no pinhole in the foil). The position and width of the injected beam were obtained by measuring the beam profile with a profile monitor placed just $56 \mathrm{~mm}$ upstream of the foil location, where the widths in standard deviation $(\sigma)$ were measured

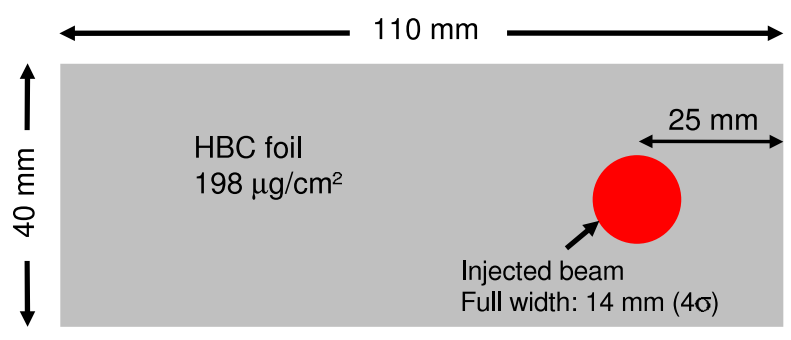

FIG. 4. The first generation primary HBC stripper foil used in the RCS was with a vertical size of $40 \mathrm{~mm}$. The injected $\mathrm{H}^{-}$beam is shown by the red circle and had a full width of $14 \mathrm{~mm}$. For the present study, the injected beam was positioned in the center of the foil in the vertical direction and the edge of the foil in the horizontal direction was set as far as $25 \mathrm{~mm}$ from the injected beam center in order to make sure for practically no missing of the injected beam in the foil.

to be about $1.75 \mathrm{~mm}$ for both the horizontal and vertical planes. Typical beam profiles measured with a low intensity beam can be found in [10].

The experiment was performed with a linac beam peak current of $15 \mathrm{~mA}$, which has been fixed for the recent user run of the RCS downstream facilities. The macropulse length of the beam was used up to the maximum of $0.5 \mathrm{~ms}$ with a chopping width of $600 \mathrm{~ns}$ and two bunches having $2.5 \times 10^{13}$ particles per bunch from the linac were injected into the RCS. Formation of the intermediate pulses by chopping the linac beam micropulses, timing relation between the linac and the RCS can be found in [9]. The measurement was done with a repetition rate of $1 \mathrm{~Hz}$, where an average of 64 shots and four sets of data for each individual setup were taken.

\section{EXPERIMENTAL MEASUREMENT}

Figure 5 shows the time domain signal of the H0CT taken in the single-pass extraction mode. Because of only a small fraction of the waste beam with realistic operation, the time domain signal of the HOCT with beam is almost identical to that with no beam and thus it is very hard to extract the real beam signal from this data in terms of both quality and quantity. However, as shown in the power spectrum in Fig. 6, an FFT analysis of the time domain signal, on the other hand, clearly identifies the beam signal corresponding to the chopping frequency, which is set to the $(h=2)$ frequency. The spectrum shown by the red color is an FFT analysis of the raw signal shown in Fig. 5, whereas the spectra shown by the black and blue colors correspond to the data taken in the injection beam dump mode (with all injected beam transported to the H0 dump) and the no beam (noise or the background level), respectively. In contrast to the no beam data, the signal corresponds to the fundamental frequency of $0.940 \mathrm{MHz}$ and successive higher order harmonics at $\mathrm{rf}$ multiples are clearly seen. It may be important to mention that the fundamental frequency of the RCS rf system with 


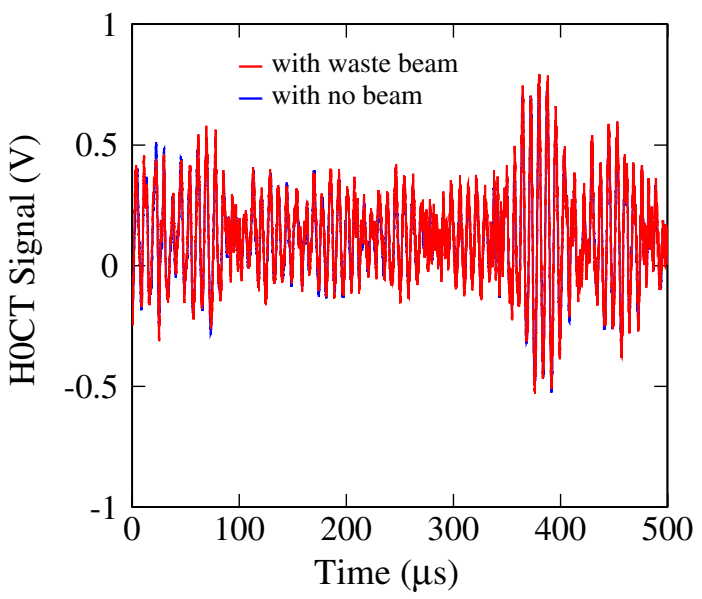

FIG. 5. Signal of the HOCT as a function of the injection time taken in the single-pass extraction mode with beam (red) and with no beam (blue) with an average of 64 shots for the foil and beam positioning as shown in Fig. 4. Data with beam was almost identical to that with no beam at this stage and thus it was hard to extract the real beam signal from this data in order to estimate the waste beam.

no momentum offset is $0.9382 \mathrm{MHz}$ but for the present operation a momentum offset $(\Delta p / p)$ of $-0.2 \%$ is set, which directly changes the chopping frequency of the linac beam. As a result, a frequency shift of $0.001295 \mathrm{MHz}$ occurs and thus the fundamental frequency is expected to be $0.9395 \mathrm{MHz}[9,11]$. The signal that corresponds to the fundamental frequency was used for the analysis.

Figure 7 shows an expanded view of the FFT spectra highlighted near the fundamental frequency region for all three cases in the upper part and only for the two cases in

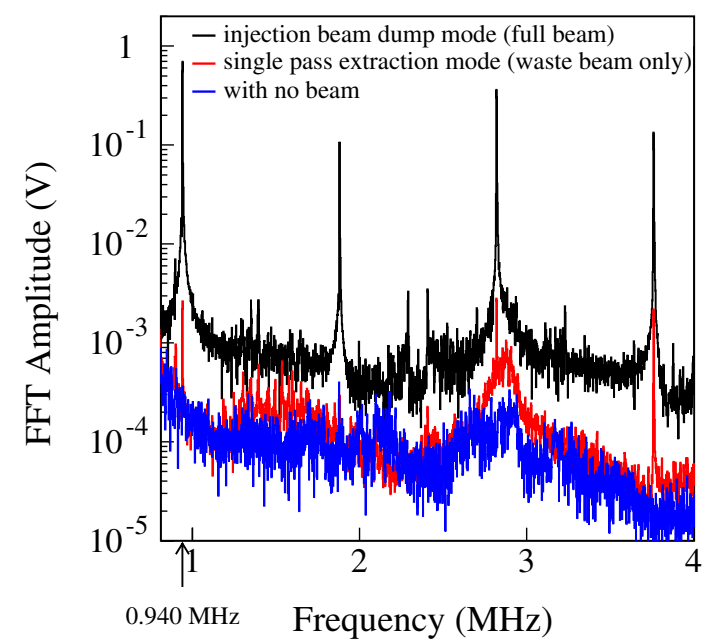

FIG. 6. Frequency spectra with an FFT analysis of the raw signal of H0CT. Data shown by the red color corresponds to the raw signal as shown in Fig. 5, whereas data shown by the black and blue colors correspond to the FFT spectrum with all of the injected beam transported to the $\mathrm{H} 0$ dump and no beam for noise level, respectively.
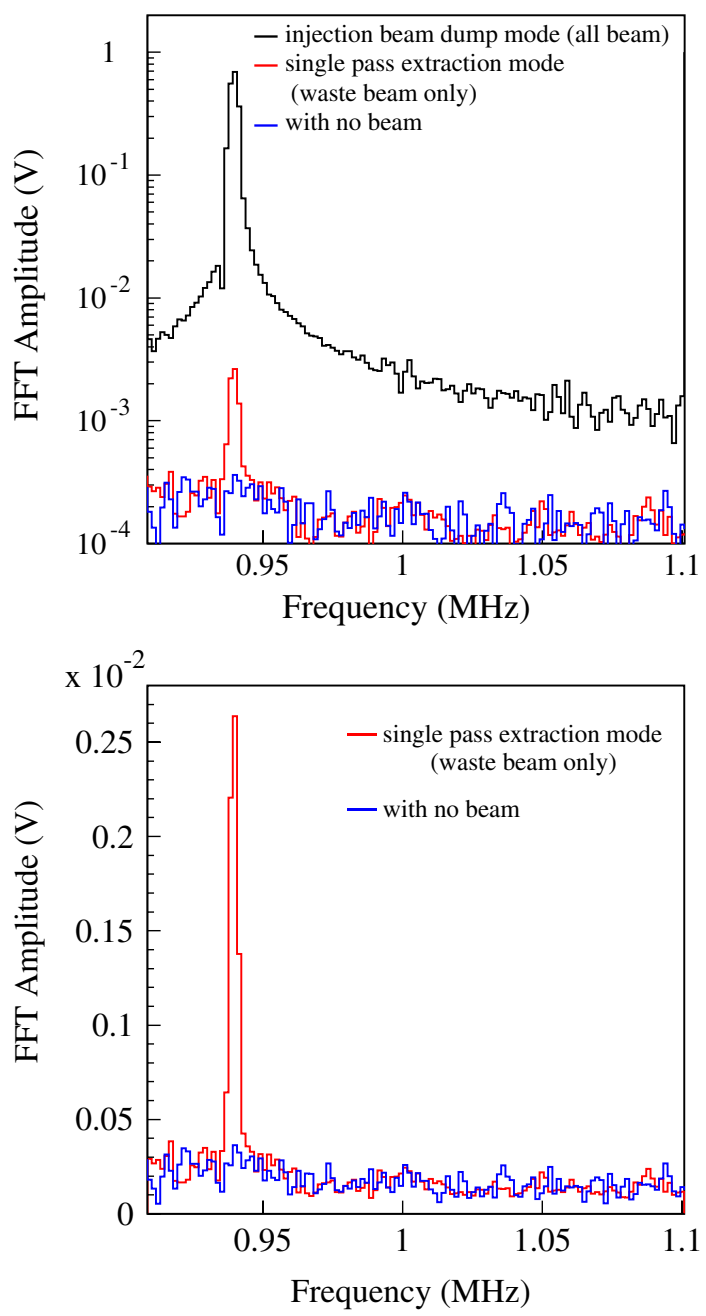

FIG. 7. Expanded view near the fundamental frequency region of the FFT spectra shown in Fig. 6. Data for all three cases are in the upper graph, whereas data for the later two cases in the lower graph are shown with a linear vertical scale. No indication of any signal in the expected frequency region with no beam as compared to a clear signal with beam can easily be seen. The signal with beam was about 1 order of magnitude higher as compared to that with no beam at the fundamental frequency of $0.940 \mathrm{MHz}$.

the lower part, respectively. The vertical axes in the upper and the lower part are shown in a logarithmic and linear scale, respectively. The difference of the signal with beam at the expected frequency region to that with no beam can easily be seen.

The FFT amplitudes corresponding to the fundamental frequency $(h=2)$ for each data are found as follows: (1) all of the injected beam to the H0 dump (black): $692.3 \pm 0.2 \mathrm{mV}$; (2) waste beam to the H0 dump (red): $2.64 \pm 0.2 \mathrm{mV}$; (3) no beam data (blue): $0.36 \pm 0.2 \mathrm{mV}$.

The peak height with a nominal setting is nearly 1 order of magnitude higher than that with no beam data. Also as explained earlier, the fraction of the waste beam at the $\mathrm{H} 0$ dump is extracted from the ratio of (2) to (1) and was found 


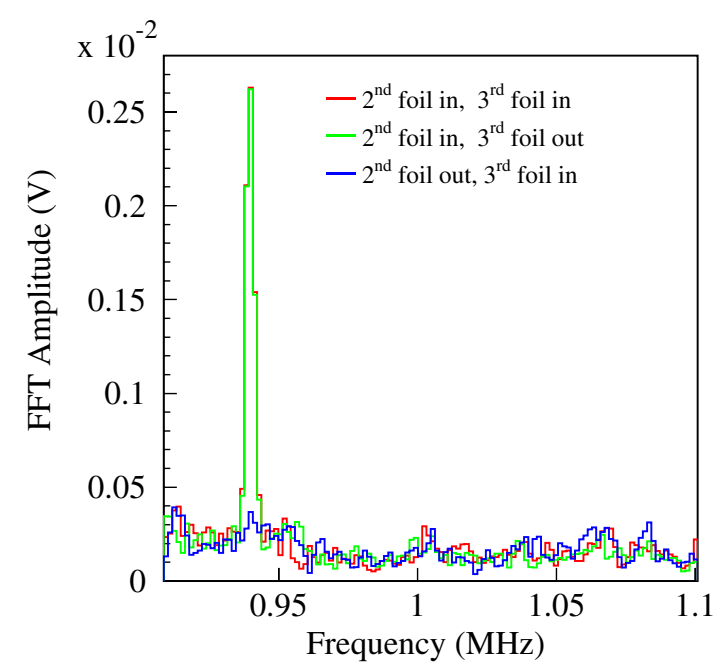

FIG. 8. Individual measurement of the $\mathrm{H}^{0}$ and $\mathrm{H}^{-}$component of the waste beam. FFT spectra for measuring separately the $\mathrm{H}^{-}$ one, the $\mathrm{H}^{0}$ one, and as a whole $\left(\mathrm{H}^{-}\right.$and $\left.\mathrm{H}^{0}\right)$ of the waste beam are shown by the blue, the green, and the red colors, respectively. Almost no difference in between the red and green colored spectra and no clear signal in the spectrum with light-blue color proves that the waste beam is mainly the partially stripped $\mathrm{H}^{0}$ one, where the unstripped $\mathrm{H}^{-}$component of the beam is negligibly small.

to be $(0.38 \pm 0.03) \%$, which is very consistent with the expectation of $0.4 \%$ as the stripper foil with a thickness of $198 \mu \mathrm{g} / \mathrm{cm}^{2}$ was used [5].

The measured $0.38 \%$ of the waste beam was mainly due to the partially stripped $\mathrm{H}^{0}$ beam at the primary stripper foil, which was further stripped to a proton beam at the $2 \mathrm{nd}$ stripper foil and transported to the injection beam dump as can be seen in Fig. 3. In order to make sure that there was no unstripped $\mathrm{H}^{-}$beam or even to measure its quantity (if any), data represented by the red color in Fig. 5 are taken by a different combination of insertion and removal of the 2nd and 3rd stripper foils.

Figure 8 shows FFT spectra for such a measurement performed in the recent study. The spectrum shown by the red color was taken in the nominal setting of all of the stripper foils [see Fig. 3(b)]. As a result, both of the partially stripped $\mathrm{H}^{0}$ and unstripped $\mathrm{H}^{-}$(if any) beams were transported to the dump through secondary stripping at the 2 nd and 3rd stripper foils, respectively. However, the spectra shown by the blue and green colors were taken by removing the 2nd and 3rd stripper foils, respectively. As a result, unstripped $\mathrm{H}^{-}$and partially stripped $\mathrm{H}^{0}$ can thus only be transported to the dump, respectively. The spectrum with green color was found to be almost similar to that with the red color, whereas the spectrum shown in the blue color was found to be very identical to that with no beam data (see Fig. 7). The small difference seen between the red and green color spectra is just within the level of the measurement error. The measurement by using the present stripper foil with a proper positioning of the injected beam on the foil thus proved that the waste beam at the injection beam dump by using the first generation stripper foil is mainly the partially stripped $\mathrm{H}^{0}$, where the unstripped $\mathrm{H}^{-}$beam is negligibly small as expected.

We have also checked the linearity of the HOCT by using beam. To do this, the RCS mode was chosen to be the circulating mode [see Fig. 3(c)]. The linearity was checked by the response of the FFT amplitude as a function of the transported beam current at the H0 dump. The beam current at the HO dump was varied by removing the primary stripper foil gradually from the beam. In the beginning, the primary stripper foil position with respect to the beam was as shown in Fig. 4 and gradually removed (right to the left) from the beam. Removal of the primary stripper foil gradually makes an increase of the injected beam missing the foil (unstripped beam) and thus gradually increasing the beam current at the $\mathrm{H} 0$ dump by reducing the circulating beam current. As mentioned earlier, the unstripped $\mathrm{H}^{-}$beam is converted to a proton beam by the 3rd stripper foil and transported to the H0 dump. The removal of the primary stripper foil far enough from the full size of the linac beam makes the situation equivalent of the injection dump mode as can be seen in Fig. 3.

For measuring the beam current at the HO dump and the circulating beam current in the ring, data were taken simultaneously by the H0CT and a direct current transformer (DCCT), respectively. The DCCT is placed in the first arc section of the RCS and is about $50 \mathrm{~m}$ downstream from the primary stripper foil [10]. The beam current measured by the DCCT at each foil position was normalized to that with initial foil position and then the fraction the beam current at the H0 dump was calculated which was further normalized to particles per two bunches.

Figure 9 shows a plot of the FFT amplitude of the fundamental frequency as a function of the beam intensity

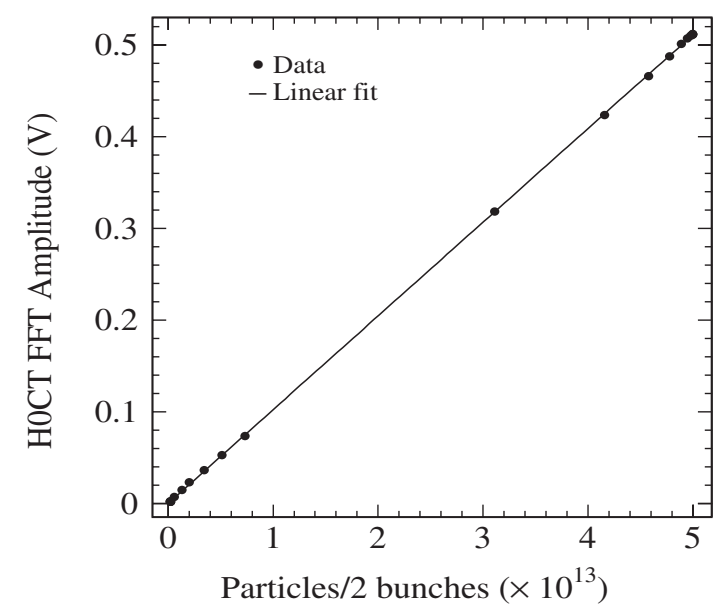

FIG. 9. FFT amplitude of the HOCT data as a function of the beam intensity. The solid circles are the measurement data for each corresponding beam intensity where the solid line is a linear fit. The linearity was found to be very good throughout the whole intensity range used in the present experiment. 
at the H0 dump. The error bars are within the size of the symbols. The solid line is linear fitting of the data and was found to be well fitted through the whole range. The linearity of the H0CT was thus found to be very good from a minimum to the maximum beam current used in the present experiment.

\section{ONLINE MONITORING}

As it has been proven that the present method can easily measure even $0.4 \%$ of the waste beam at the H0 dump, we have started using this method for online monitoring of the waste beam intensity during the RCS operation. It is also found to be very useful and efficient for a proper positioning with a new foil with different size as well as for new injection parameters.

In contrast to the study (see Fig. 4), the primary stripper foil position with respect to the beam was optimized as shown in Fig. 10. This was needed in order to keep the circulating beam hitting probability below an acceptable amount so as to reduce the uncontrolled beam loss near the injection area due to the multiple Coulomb scattering of the beam at the foil [12]. The foil edge in the horizontal direction was set $7 \mathrm{~mm}$ far from the injected beam center, where an offset of $-10 \mathrm{~mm}$ was set in the vertical direction. The fraction of the waste beam in this case is the ratio of the HOCT signals with this circulating mode and the injection beam dump mode [see Figs. 3(c) and 3(c)].

Figure 11 shows a trend of the HOCT signal for more than a week taken in run 33 during RCS user operation to the MLF. An average beam power of $120 \mathrm{~kW}$ at the extraction energy of $3 \mathrm{GeV}$ was delivered to the MLF, which corresponds to about $2 \times 10^{13}$ particles per two bunches in one accelerator cycle of $40 \mathrm{~ms}$. The injected beam current was measured by the linac slow current transformer placed just a few meters upstream of the RCS injection point (primary stripper foil) and normalized to particles per two bunches as shown by the data with black color. Normalizing the H0CT FFT signal by the injected beam current, the fraction of the waste beam in

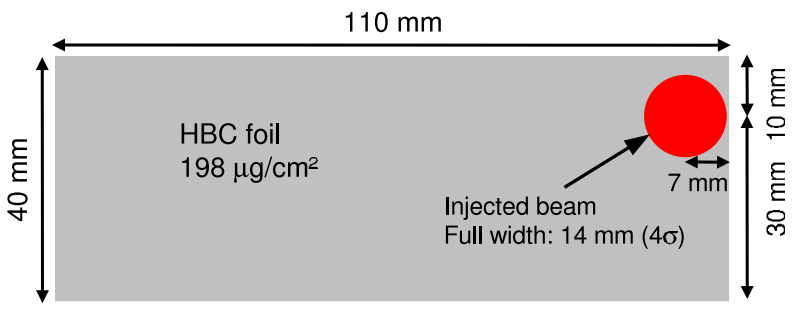

FIG. 10. The positioning of first generation primary stripper foil with respect to the injected beam for the RCS operation in run 33. Injected beam center to the foil edge was $7 \mathrm{~mm}$ in the horizontal direction, whereas the foil was with an offset of $-10 \mathrm{~mm}$ in the vertical direction in order to reduce the beam loss due to the multiple Coulomb scattering of the circulating beam at the foil.

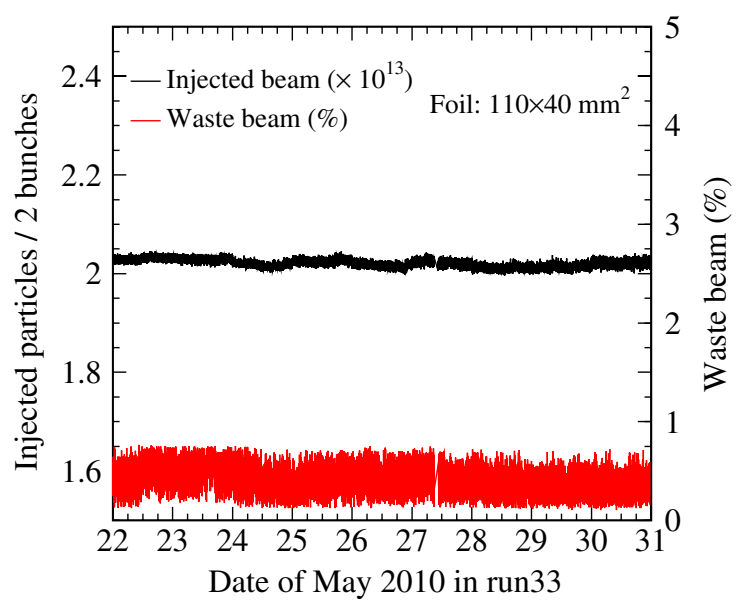

FIG. 11. A trend of the H0CT signal for about a week taken during RCS user operation for the MLF in run 33. The position of the primary stripper foil and the injected beam was optimized in such a way that the waste beam at the H0 dump was found to be as expected of $0.4 \%$ and there was no significant change of the quantity throughout the week.

the H0 dump was calculated as shown by the data with red color. The waste beam at the H0 dump was as expected near $0.4 \%$ and there was no significant change of the fraction of the waste beam throughout the week.

However, in the summer shutdown of 2010, we prepared our second generation of the primary stripper foils. The horizontal size was the same with $110 \mathrm{~mm}$ and the change was in the vertical size. Namely, two foils with dimensions of $110 \times 15 \mathrm{~mm}^{2}$ and $110 \times 20 \mathrm{~mm}^{2}$ were installed. The vertical size of the foil was needed to be optimized in order to reduce the uncontrolled beam loss as much as possible caused by the foil scattering in the injection area [12]. Because of the transverse painting scheme in the RCS, the circulating beam goes away from the foil as a function of the painting injection time and thus the vertical size of the foil is the main concern for the foil scattering beam loss with the present configuration of the foil dimension $[3,12]$. The injected beam full size until the summer shutdown was $14 \mathrm{~mm}$ (see Fig. 10) and thus a vertical size of $15 \mathrm{~mm}$ was thought to be enough for the RCS operation at the present beam power especially, with the present peak current of the linac beam. The transverse emittance of the injected beam usually increases with higher peak current as also found in the linac beam study [13]. On the other hand, a bending effect of the foil edge with a high power beam also needs a margin to consider in determining the size of the stripper foil.

These new foils were first used in the very recent operation of the RCS. Figure 12 shows those two foils with injected beam plotted together. It is important to mention that, due to insufficient tuning of the linac beam, the transverse profile of the beam was much wider as well as the beam tail part component was much higher as compared to the previous runs. The full width of the beam was 

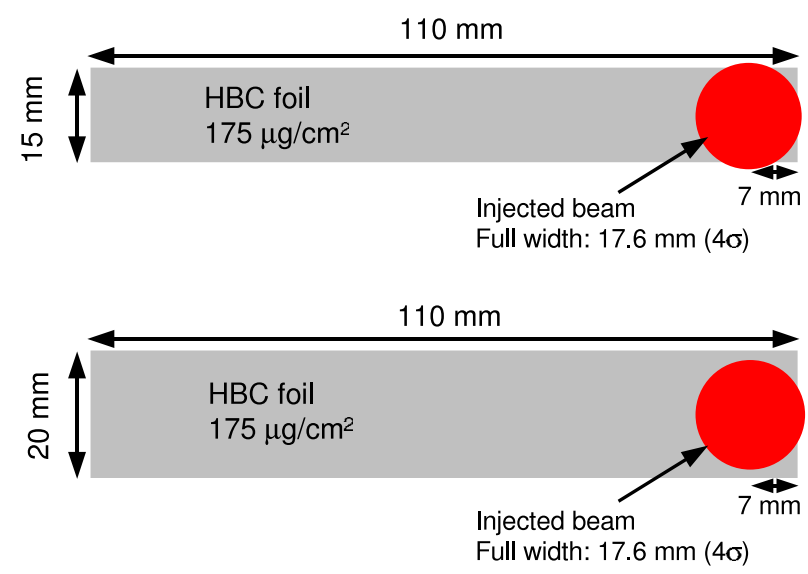

FIG. 12. The second generation primary stripper foils having smaller vertical sizes of $15 \mathrm{~mm}$ (upper) and $20 \mathrm{~mm}$ (lower) plotted together with injected beam positioning for the RCS operation in run 36. The foil edge from the injected beam center kept the usual setting of $7 \mathrm{~mm}$ in the horizontal direction with no offset of the foil in the vertical direction.

measured to be $17.6 \mathrm{~mm}$ as compared to $14 \mathrm{~mm}$ measured in the previous runs. The foil edge from the injected beam center in the horizontal direction was set as usual to $7 \mathrm{~mm}$ and with no offset in the vertical direction. As a result, tail parts of the beam in both horizontal and vertical directions and at least in the horizontal direction missed hitting the foil with vertical size of 15 and $20 \mathrm{~mm}$, respectively.

Figure 13 shows a trend of the H0CT for two cycles of RCS operation in run 36 with the same beam power of

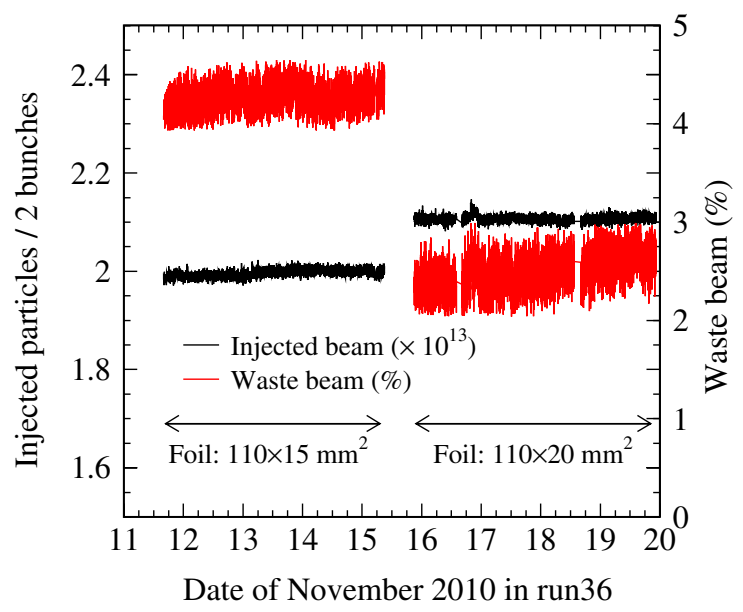

FIG. 13. A trend of the H0CT signal for two cycles in run 36 by using second generation stripper foils with reduced vertical sizes. Mostly because of the unexpected long tails of the injected beam, the waste beam at the H0 dump by using a vertical foil size of $15 \mathrm{~mm}$ in the first cycle was found to be an order of magnitude higher than expected as well as observed so far by using the first generation stripper foil. It was then exchanged by a vertical size of $20 \mathrm{~mm}$ which could reduce the waste beam by nearly a half but it was still a few times higher than the expected level.
$120 \mathrm{~kW}$ to the MLF. In the first cycle the vertical foil size of $15 \mathrm{~mm}$ was used, where the fraction of the waste beam at the H0 dump was measured more than $4 \%$ and was thus about an order of magnitude higher than the measured value in the previous runs by using the first generation foil (see Fig. 11). The extracted beam power to the MLF was thus found to be about $4 \%$ lower as compared to the previous $120 \mathrm{~kW}$ operations. A routine residual radiation survey was performed in the scheduled maintenance day of the 15th of November, 2010. The residual radiation at the entrance of the $\mathrm{H} 0$ dump was found to be more than 1 order of magnitude higher as compared to previous runs measured as usual with a time delay ( $>4 \mathrm{~h}$ ) after the beam stop. Thus, the residual radiation measurement is consistent with the monitoring by the H0CT.

The foil was then changed to a vertical size of $20 \mathrm{~mm}$ for the remaining days of the running cycle. Injected beam was expected to be covered fully by the foil in the vertical direction. Naturally, waste beam at the H0 dump was found to be reduced significantly but it was still about $2.5 \%$. It is important to mention that the thickness of these new foils was measured to be much lower than that of the first generation foil: about $175 \mu \mathrm{g} / \mathrm{cm}^{2}$. As a result, partial stripped $\mathrm{H}^{0}$ was found to be nearly 2.5 times higher as compared to the first generation foil: about $1 \%$ [5]. The unstripped $\mathrm{H}^{-}$component of the waste beam due to the injected beam misses the foil in both horizontal and vertical directions, pinholes in the foil (if any) remain of about $3 \%$ and $1.5 \%$ with the vertical foil size of 15 and $20 \mathrm{~mm}$, respectively. The trend of the waste beam however was stable during the whole cycle and thus stability of the injected beam as well as no deformation of the foil itself can also be understood from this online data.

\section{SUMMARY}

The FFT analysis on the very low signal made it possible to measure $0.4 \%$ of the waste beam with good accuracy at the injection beam dump of the RCS of J-PARC. The absolute error of the monitor system is $0.2 \mathrm{mV}$, which gives the ratio of the waste beam to the injected beam as $(0.38 \pm$ $0.03) \%$. The present method has already been successfully used in optimizing the foil parameters as well as online monitoring of the waste beam during the user operation. One of the main applications of this study is to determine the foil lifetime at the high power operation. The stripper foil has a certain lifetime and this is always a big concern for high power operation. Foil degradations due to radiation and the associated high temperature rise cause an increase of the waste beam. The injection dump, however, has a limited capacity of only $4 \mathrm{~kW}$. With such an accurate monitoring system, the time to replace the stripper foil can precisely be determined before the power of the waste beam at the injection dump reaches the limit. In a high power machine where a charge-exchange technique is used, a stripping efficiency is aimed as high as nearly 
$100 \%$ and thus ideally a very small amount of the waste beam is expected. The capacity of the waste beam dump is then determined accordingly. However, due to slightly changed operational conditions and parameters as well (like in RCS today), the waste beam might increase. A precise and useful technique for monitoring the waste beam is thus very important. Hence, the present idea is believed to be applicable not only in the RCS of J-PARC but also in any other similar high power machines.

\section{ACKNOWLEDGMENTS}

The authors are grateful to all of the members of the RCS beam commissioning team for their support during the experiment. Helpful discussions with Dr. Fumihiko Tamura of J-PARC about RCS rf system together with the chopping system are highly appreciated. It is also a great opportunity for the authors to acknowledge the effort of Dr. Isao Sugai from KEK for his continuous support in preparing many different sizes of the HBC stripper foils for the RCS operation from the very early stages to the present. The authors are also grateful to Dr. Alexander Schnase of J-PARC for technical comments and proof reading.

[1] M. Kinsho, in Proceedings of the First International Particle Accelerator Conference (IPAC 10), Kyoto, Japan (2010), pp. 627-629.

[2] High-Intensity Proton Accelerator Project Team, JAERI Technical Report No. 2003-044, 2003.
[3] P. K. Saha et al., Phys. Rev. ST Accel. Beams 12, 040403 (2009).

[4] I. Sugai, Y. Takeda, M. Oyaizu, H. Kawakami, Y. Irie, A. Takagi, H. Hattori, and K. Kawasaki, Nucl. Instrum. Methods Phys. Res., Sect. A 613, 457 (2010).

[5] R. C. Webber and C. Hojvat, IEEE Trans. Nucl. Sci. 26, 4012 (1979).

[6] W. Chou, M. Kostin, and Z. Tang, Nucl. Instrum. Methods Phys. Res., Sect. A 590, 1 (2008).

[7] P. K. Saha, H. Hotchi, H. Harada, M. Yoshimoto, K. Yamamoto, Y. Yamazaki, and I. Sugai, in Proceedings of the 1st International Particle Accelerator Conference IPAC0'10 (Ref. [1]), pp. 3921-3923.

[8] K. Satou, N. Hayashi, H. Hotchi, Y. Irie, M. Kinsho, M. Kuramochi, P. K. Saha, Y. Yamazaki, and S. Lee, in Proceedings of the 2007 Particle Accelerator Conference, Albuquerque, New Mexico (IEEE, New York, 2007), pp. 4084-4086.

[9] M. Yamamoto et al., Nucl. Instrum. Methods Phys. Res., Sect. A 621, 15 (2010).

[10] H. Hotchi et al., Phys. Rev. ST Accel. Beams 12, 040402 (2009).

[11] F. Tamura et al., Phys. Rev. ST Accel. Beams 12, 041001 (2009).

[12] P. K. Saha, in Proceedings of the 46th ICFA Advanced Beam Dynamics Workshop (HB2010), Morschach, Switzerland (2010), pp. 324-328.

[13] M. Ikegami, H. Sako, A. Miura, and G. Wei, in Proceedings of 46th ICFA Advanced Beam Dynamics Workshop (HB2010), Morschach, Switzerland (Ref. [12]), pp. 548-550. 